Int. J. Dev. Biol. 57: 767-772 (2013)

doi: $10.1387 / \mathrm{ijdb} .120234 \mathrm{tw}$

\title{
Identification of promoter elements responsible for gonad- specific expression of zebrafish Deadend and its application to ovarian germ cell derivation
}

\author{
TEN-TSAO WONG*, ABRAHAM TESFAMICHAEL and PAUL COLLODI \\ Department of Animal Sciences, Purdue University, West Lafayette, Indiana, USA
}

\begin{abstract}
We discovered that a 150-bp region of zebrafish deadend (dnd) spanning the translation start codon, exon 1 and part of intron 1 is required to direct heterologous neomycin-resistance gene (neo) expression specifically in the gonad, similar to endogenous $d n d$. Using an 8.3-kb dnd promoter that contains this $\mathbf{1 5 0}$-bp region, we generated $\mathbf{T g}(d n d: n e o-d n d)$ transgenic zebrafish in which the expression of Neo was detected specifically in ovarian germ cells. The transgenic fish were used to initiate primary ovarian germ cell cultures with antibiotic G418 to select ovarian germ cells and eliminate ovarian somatic cells. RT-PCR results demonstrated that the drug-selected ovarian germ cells continued to express germ-cell markers nanos3, vasa and dnd. Growth assays demonstrated that recombinant zebrafish Lif had a significant mitogenic effect on the ovarian germ cells. When long-term ovarian germ cell cultures were transplanted into two-week-old infertile larvae, they successfully colonized and directed the formation of a truncated gonad in the recipient adult fish. Histological examination of the recipient adult fish revealed that 9 out of 34 individuals $(26 \%)$ possessed donor-derived cells in their gonads. The identification of zebrafish dnd promoter and the use of this promoter to generate $\mathrm{Tg}(\mathrm{dnd}$ :neo-dnd) led to the success of germ cell isolation through drug selection to generate homogenous germ cells that can be used to study zebrafish germ cell biology and may lead to a cell-mediated gene transfer strategy.
\end{abstract}

KEY WORDS: zebrafish, deadend, ovarian germ cell, transplantation

\section{Introduction}

Deadend (Dnd) is an evolutionary conserved RNA-binding protein that specifically expresses in germ cells and plays a critical role in germ cell development during embryogenesis in vertebrates (Horvay et al., 2006, Weidinger et al., 2003, Youngren et al., 2005). In zebrafish embryos, knockdown of Dnd resulted in the failure of primordial germ cells (PGCs) to acquire motility. As such PGCs were restricted to the deep blastoderm and died by the end of the first day of development (Weidinger et al., 2003). In Xenopus embryos, knockdown of Dnd caused a depletion of PGCs at tadpole stages of development (Horvay et al., 2006). In mouse, mutations of Dnd1 (known as Ter) locus resulted in germ cell loss and increased vulnerability to spontaneous testicular germ-cell tumors (Youngren et al., 2005).

In this study, we describe the identification of zebrafish dnd promoter. We found that a $150 \mathrm{bp}$ region covering the translation start codon, exon 1 and part of intron 1 is required for restricting dnd expression to be gonad-specific. An 8.3-kb dnd promoter that contains this 150-bp region was used to generate $\mathrm{Tg}$ (dnd:neo-dnd) transgenic line that expresses neo in germ cells. Methods were established for drug selection and in vitro propagation of the ovarian germ cells derived from the $T g(d n d: n e o-d n d)$. The culture condition was further optimized using recombinant zebrafish leukemia inhibitory factor (Lif). The ability to isolate zebrafish homogenous ovarian germ cells and propagate the cells in culture provides a strategy towards developing a cell-mediated gene targeting approach in this model system.

Abbreviations used in this paper: Dnd, Deadend; Lif, leukemia inhibitory factor; Neo, neomycin-resistance gene; Pac, puromycin-resistance gene; PGC, primordial germ cell.

\footnotetext{
*Address correspondence to: Ten-Tsao Wong. Department of Animal Sciences, Purdue University, 901 W. State Street, West Lafayette, IN 47907 USA. Tel: +1-(765)-494-8015. Fax: +1-(765)-494-6816. E-mail:Wong20@ purdue.edu
} 


\section{Results}

\section{A 150-bp region spanning dnd exon 1 and intron 1 is required for tissue-specific expression of the dnd-neo transgene}

In an effort to identify the promoter elements responsible for dnd expression, we first cloned an 8.15-kb 5' flanking genomic fragment that contained the first exon up to the first nucleotide of the translation initiation codon and a 0.98-kb 3' genomic fragment that contained the $d n d$ translation stop codon and 3' untranslated region (UTR). This construct (dnd ${ }^{p 8.15}$-neo-dnd3'UTR; Fig. 1A) was used to generate a transgenic line and the tissue distribution of endogenous dnd mRNA (Fig. 1B) and dnd-neo mRNA (Fig. $1 \mathrm{C})$ was compared by RT-PCR. The results revealed that unlike endogenous dnd that was only detected in the ovary and testis, $d n d-n e o$ was detected in all tissues examined. When a transgenic line was generated using a construct in which the length of the promoter region was extended to $8.30 \mathrm{~kb}$ at the 3' flanking region of the promoter to include the entire exon 1 and $109 \mathrm{bp}$ of intron 1 (dnd ${ }^{p 8.30}-n e o-d n d 3^{\prime} U T R$, Fig. 1D), expression of $d n d$-neo in this transgenic line was observed specifically in the ovary and testis, similar to the endogenous $d n d$ (Fig. 1E). The transgenic line of fish carrying $d n d^{108.30}-$ neo-dnd3'UTR is referred to as $T g(d n d: n e o-$ dnd). Promoter analysis revealed that the additional 150 bp region contains the consensus nucleotide sequences for transcription A

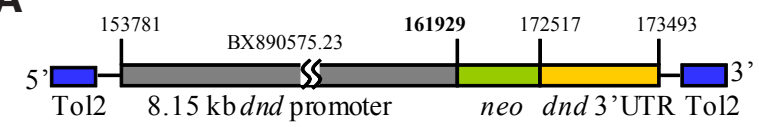

B

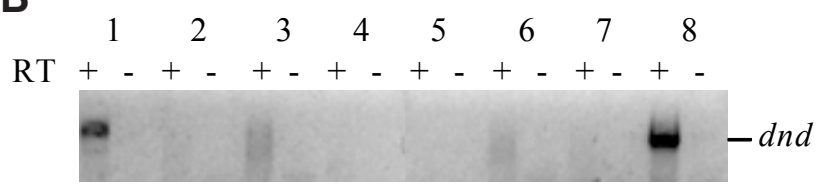

C

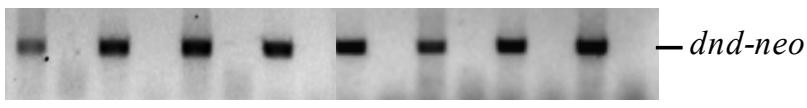

D

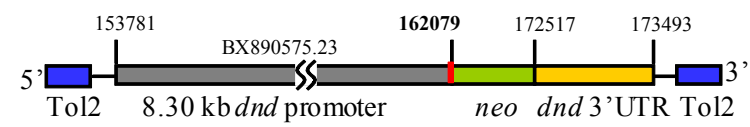

E

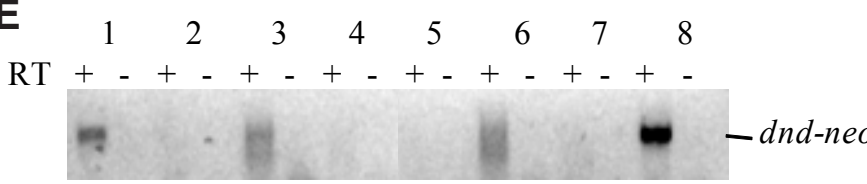

Fig. 1 (left). Identification of the dnd promoter for generating Tg(dnd:neo-dnd) transgenic fish. (A) Diagram of the transgenic construct using an $8.15 \mathrm{~kb}$ dnd promoter that contained exon 1 up to the first nucleotide of translation initiation codon and a $0.98 \mathrm{~kb}$ dnd $3^{\prime} U T R$ and flanking region. (B) Tissue distribution of endogenous dnd mRNA. (C) Tissue distribution of dnd-neo mRNA in transgenic fish derived from dnd ${ }^{\text {p.15 }}$-neo-dnd3'UTR construct. (D) Diagram of the transgenic construct using an $8.30 \mathrm{~kb}$ dnd promoter that contained the addition $150 \mathrm{bp}$ (red) to include the entire exon 1 and a part of intron 1 and a $0.98 \mathrm{~kb}$ dnd $3^{\prime}$ UTR and flanking region. (E) Tissue distribution of dnd-neo mRNA in transgenic fish derived from dnd ${ }^{\mathrm{p} 8.30}$-neodnd3'UTR construct. Tissues used in RT-PCR: Testis(1); Gastrointestinal tract(2); Brain(3); Gill(4); Liver(5); Heart(6); Muscle(7); Ovary(8).

Fig. 2 (right). Transcription binding site analysis of the $\mathbf{1 5 0}$ bp (red) that contains gonad-specific expression elements of dnd. Transcription factor (E47), myc common transcription factor 1 (myc-CF1), activator protein 2 (AP-2), lymphoid transcription factor (LyF-1), epidermal growth factor receptor-specific transcription factor (ETF), TATA box binding protein (TBP), glucocorticoid receptor (GR) and T cell-specific transcription factor (TCF-1).

\section{RESULTS FROM THREE TRANSPLANTATION (T) EXPERIMENTS USING CULTURED OVARIAN GERM CELLS INTRODUCED INTO TWO-WEEK-OLD STERILE RECIPIENTS}

\begin{tabular}{|c|c|c|c|c|}
\hline $\begin{array}{l}\text { Exp. } \\
\text { groups }\end{array}$ & $\begin{array}{l}\text { Number of } \\
\text { recipients } \\
\text { transplanted }\end{array}$ & $\begin{array}{l}\text { Number and (\%) of } \\
\text { recipients survived to } \\
4 \text { weeks }\end{array}$ & $\begin{array}{c}\text { Number and (\%) } \\
\text { of recipients carrying } \\
\text { ovarian } \\
\text { germ cells } \\
\text { in gonadal region }\end{array}$ & $\begin{array}{l}\text { Number } \\
\text { of recipients } \\
\text { surviving } \\
\text { to adulthood }\end{array}$ \\
\hline$\overline{\mathrm{T} 1}$ & 33 & 27 & $9(33 \%)$ & 24 \\
\hline T2 & 28 & 19 & $9(47 \%)$ & 17 \\
\hline T3 & 47 & 30 & $11(36 \%)$ & 24 \\
\hline Total & 108 & 76 (70.4\%) & $29(38 \%)$ & 65 \\
\hline
\end{tabular}

factor E47, myc-CF1, AP-2, LyF-1, EGFR-specific transcription factor ETF, TBP, GR and TCF-1 (Fig. 2).

\section{Ovarian germ cell cultures derived from $\mathrm{Tg}$ (dnd:neo-dnd) fish}

Immunocytochemical analysis of ovarian tissue of $\mathrm{Tg}$ (dnd:neodnd) fish showed that Neo as well as germ-cell marker Vasa were detected in ovarian germ cells including oogonia (Fig. 3 $A, B, C)$. Therefore, a drug selection approach was developed to derive ovarian germ cell cultures initiated from the ovaries of the $\mathrm{Tg}$ (dnd:neo-dnd) fish in the presence of antibiotic G418 to select ovarian germ cells and eliminate ovarian somatic cells. In the first six weeks, the cultured ovarian germ cells were passed every 2 weeks. Thereafter, the resulting drug-resistant colonies were maintained and expanded (one passage/week) for 6 months with the estimated doubling time as 5 to 7 days at $22^{\circ} \mathrm{C}$. The drug-selected ovarian germ cells are round and about $10 \mu \mathrm{m}$ in diameter (Fig. $4 \mathrm{~A})$. To visualize the ovarian germ cells, cultures were transfected with a plasmid encoding the puromycin resistant gene and DsRed fusion protein (Pac-DsRed) driven by a CMV promoter. Following selection in antibiotic G418 and puromycin, colonies were established and expanded (Fig. 4 B,C). To optimize the culture condition for the ovarian germ cells, the growth promoting effect of recombinant zebrafish Lif was studied. Zebrafish Lif was found to have a mitogenic effect on the ovarian germ cells. The number of the ovarian germ cells increased $100 \%$ over 6 days in culture

(BX890575.23)

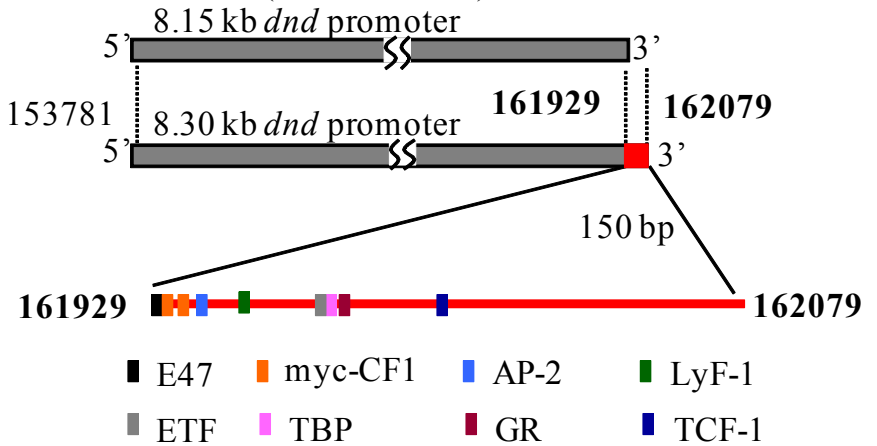



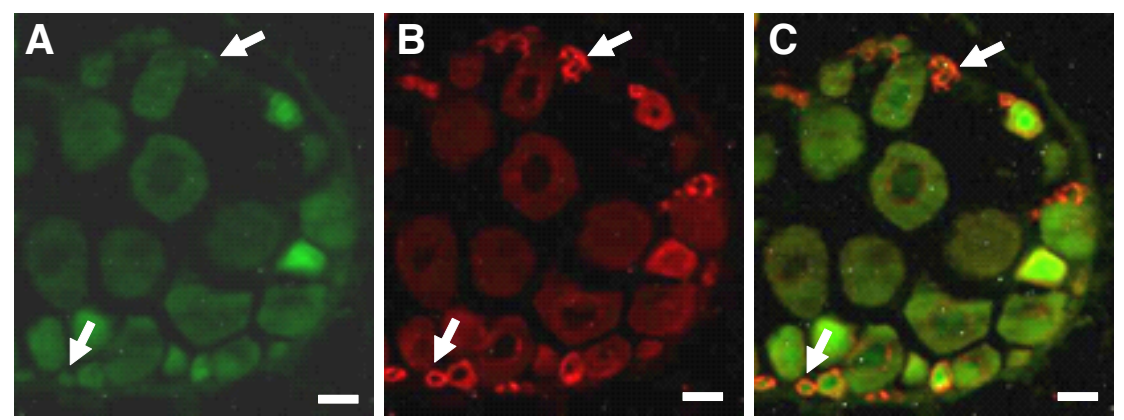

Fig. 3. Immunocytochemical analysis of ovarian tissue in Tg(dnd:neo-dnd) fish. Photomicrograph showing (A) the expression of Neo (green), (B) the expression of Vasa (red) in ovarian germ cells including oogonia (white arrows) and (C) the merge photo of $(A, B$.) Scale bar, $20 \mu \mathrm{m}$.

(Fig. 4D). RT-PCR analysis revealed that the ovarian germ cell cultures with RTS34st-Lif feeder continued to express germ-cell specific markers including nanos3, vasa and dnd for more than 6 months in culture (Fig. 4E).

\section{Long-term ovarian germ cell cultures were able to colonize the gonad after transplantation into recipient fish}

Cell transplantation experiments were performed to evaluate the capacity of the cultured ovarian germ cells to contribute to the germ line of a host fish. The G418 and puromycin selected colonies that also expressed DsRed (Fig. 4C) were used for transplantation into the abdominal cavity of infertile recipients (Fig. 5A) treated as embryos with $d$ nd antisense morpholino to block germ cell formation (Slanchev et al., 2005). Four weeks after transplantation, the recipients were examined for the presence of transplanted (DsRed-
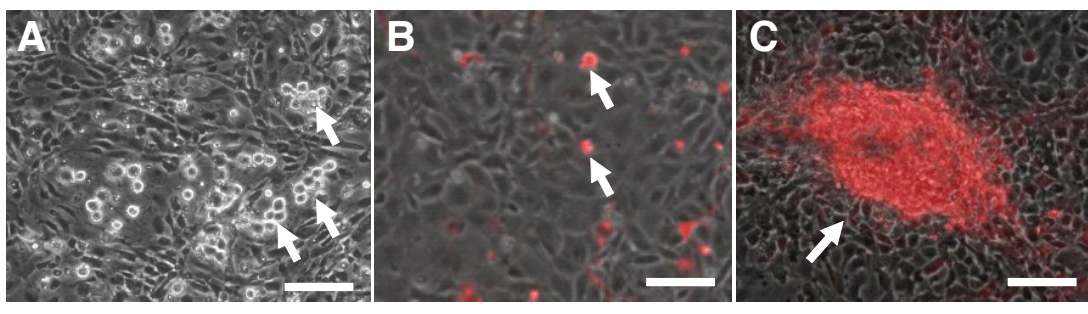

D

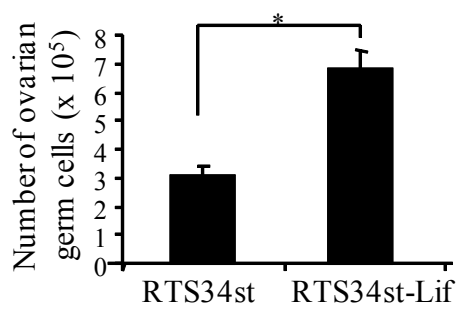

$\mathbf{E}$

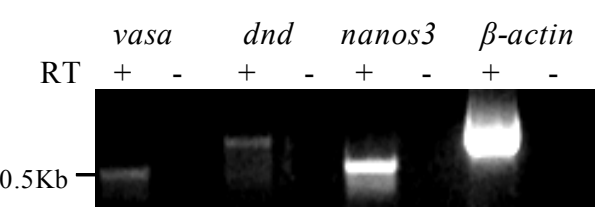

Feeder cells

Fig. 4. Ovarian germ cell cultures derived from $\mathrm{Tg}(\mathrm{dnd}$ :neo-dnd) transgenic zebrafish. Photomicrograph showing (A) the antibiotic G418 selected ovarian germ cells (arrows) grown on growth-arrested and G418-resistant RTS34st feeder cells, (B) the puromycin selected and DsRed-positive ovarian germ cells (arrows), and (C) the DsRed-positive cells grow and form an aggregate (arrow) on top of the feeder cells. (D) Zebrafish Lif significantly $(p<0.05)$ promoted the growth of ovarian germ cells in a 6-day culture. (E) RT-PCR showing that long-term cultured ovarian germ cells expressed germ-cell-specific maker genes, nanos3, vasa and dnd. *: indicates a significant difference by Student t tests. Scale bar, $50 \mu \mathrm{m}$. positive) cells. From three different transplant experiments, a total of 76 recipients were examined and $29(38 \%)$ individuals that possessed DsRed-positive cells in the gonadal region were identified (Table 1). Examination of the three fish revealed that the transplanted ovarian germ cells had colonized the gonads of the recipients and proliferated based on the presence of DsRed-positive cells in the tissue (Fig. 5B). From the three transplantations, All 65 recipients raised to adults were found to be male. Each individual was bred pairwise with at least two different wild-type female mates for over two weeks; none of the chimeric fish was able to produce fertilized eggs. Histological examination revealed that 9 out of 34 recipients (26\%) possessed DsRed-positive cells located in a truncated gonad (Fig. 5 C,D). In contrast to the non-transplanted infertile adults that possessed only thin connective tissue surrounded by adipocytes in the gonadal region (Fig. 5E), the long-term drug-selected ovarian germ cells were able to direct the formation of a truncated gonad but not any other tissue in the recipient after transplantation. Even though no sign of gametogenesis (Fig. 5F) similar to wild-type adults (Fig. 5 $\mathrm{G}, \mathrm{H}$ ) was detected in the chimeric individuals, the chimeras did

\section{Discussion}

In this study, an 8.3-kb fragment of the $d$ nd promoter was identified to drive specific neo expression in ovarian germ cells of the transgenic line. In this promoter, the elements located in the very last 150-bp region play an important role in controlling gonad-specific expression of $d n d$. Without this 150-bp region, dnd-neo mRNA was detected in all the tissues examined. Inclusion of the $150 \mathrm{bp}$ region results in the presence of the transcription factor E47 binding site being located at the translation start codon. E47 has been shown to restrict gene expression in other cell types (Yang etal., 2004) and it may play a similar role in controlling tissue-specific expression of zebrafish $d n d$.

The ovarian germ cell cultures derived from $T g$ (dnd:neo-dnd) continue to express nanos3, dnd and vasa for more than 6 months in culture. This culture system provides an in vitro platform to investigate the growth and differentiation factors that regulate ovarian germ cell development. Specific growth factor such as LIF has been added into medium to improve spermatogonial stem cell culture (Kanatsu-Shinohara etal., 2003) and ovarian germline stem cell culture (Zou et al., 2009) in mouse. Therefore, we cloned zebrafish lif and expressed it in RTS34st cells. Knowledge of Lif in zebrafish is very limited. Mopholino knockdown experiments indicated that Lif receptor $b$ (Lifrb) but not Lif is required for proper neural development (Hanington et al., 2008). Over-expression of Lif in PGCs resulted in a disruption of PGC migration through Lifra signal pathway (Wong and Collodi, 2013). In this study, we 
demonstrated that zebrafish Lif significantly promotes the growth of ovarian germ cells.

Although spermatogonial stem cell cultures have been established in fish (Hong et al., 2004, Kawasaki et al., 2012, Sakai, 2002), no selection methods are currently available to isolate homogenous germ cells. The identification of zebrafish $d n d$ promoter and the use of this promoter to generate $\mathrm{Tg}(\mathrm{dnd}$ :neo- $d n d)$ led to the success of the germ cell isolation through a long-term drug selection to eliminate somatic cells. Under long-term culture, ovarian germ cells derived from $\mathrm{Tg}$ (dnd:neo-dnd) continuously express germcell markers, dnd, vasa and nonos 3 and were able to direct gonad formation in infertile recipients. The fact that cultured ovarian germ cells were able to be specifically attracted, interact and integrate with recipient's gonadal somatic tissue, but not any other tissues,

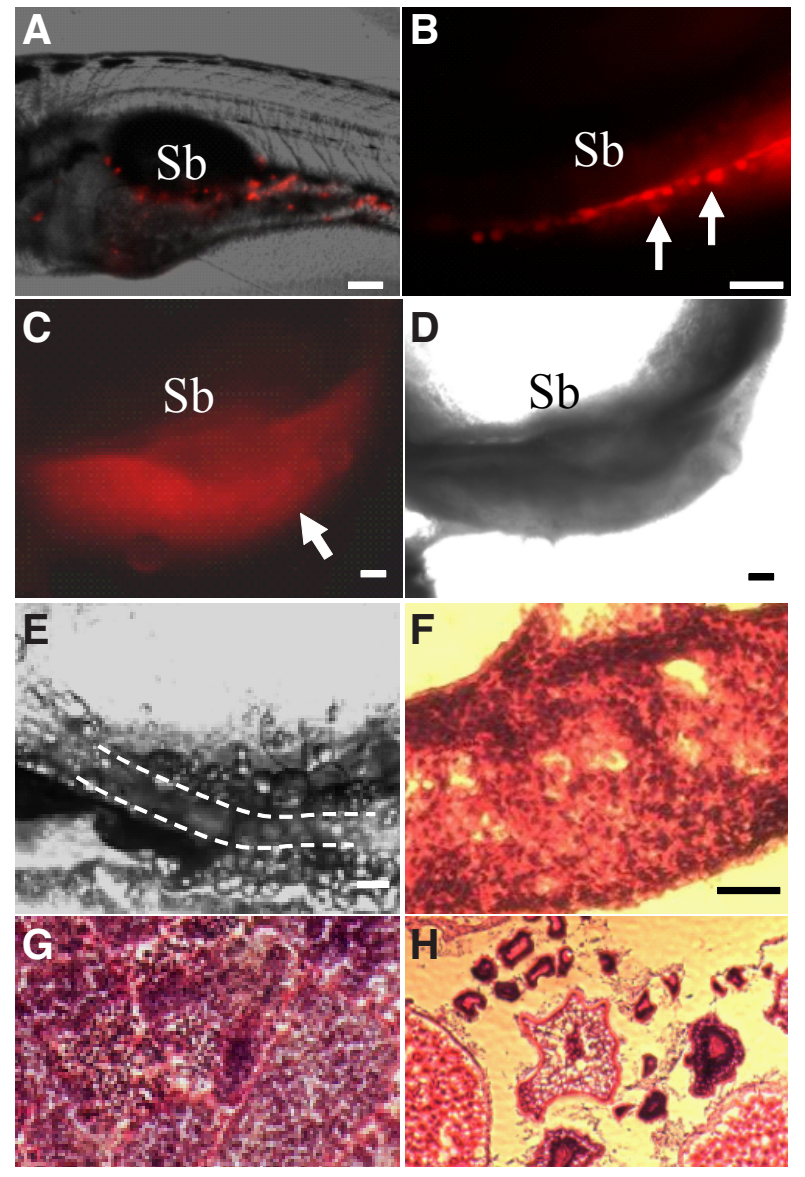

Fig. 5. Colonization and proliferation of cultured ovarian germ cells in a recipient's gonad after transplantation. (A) The transplanted ovarian germ cells (red) in the abdominal cavity of a recipient, and (B) the incorporation of transplanted ovarian germ cells (red) into the gonad tissue (arrows) under swim bladder (Sb) of a recipient larva four weeks after transplantation. (C) Fluorescence photomicrograph of a recipient gonad 3 months after transplantation showing that the transplanted ovarian germ cells (red) have proliferated and directed the formation of gonadal tissue; the arrow points to gonadal tissue; (D) the bright field image of $C$ showing the gonadal tissue. (E) In non-transplanted infertile host, only a thin tissue (indicated by dot lines) surrounded by adipocytes was found. (F) No sign of gametogenesis in adult recipients that carried transplanted ovarian germ cell, which is different from $(\mathbf{G})$ the testis and $(\mathbf{H})$ the ovary of the adult fish. Scale bar, $200 \mu \mathrm{m}$ for $(A, C, D, E)$ and $50 \mu \mathrm{m}$ for $(B, F, G, H)$ to develop into truncated gonads indicating the long-term cultured ovarian germ cells maintained certain germ cell characteristics. Our current culture system may not sufficient enough to maintain full germline distinctiveness of cultured ovarian germ cells. Others such as the chromosome abnormality may also happen during a long-term culture, which may become an obstacle that blocks meiosis. This culture system provides an initial step for searching and studying the proliferation and maintenance of zebrafish ovarian germ cells in culture which is valuable in understanding ovarian germline stem cell biology and identifying crucial growth factors for their self-renewal, stemness and proliferation. Together with germ cell transplantation technology that we have developed (Wong et al., 2011), our system is useful for future success in establishing germline-competent ovarian germline stem cell cultures.

\section{Materials and Methods}

\section{Plasmid construction}

The dnd genomic sequence was identified in zebrafish DNA sequence BX890575.23. Primers were designed to amplify an $8.15-\mathrm{kb}$ fragment that contained exon 1 up to the first nucleotide of translation initiation codon (nucleotides 153781 to 161929 in BX890575.23) and an 8.30-kb fragment that contained the entire exon 1 and a part of intron 1 (nucleotides 153781 to 162079 ). The following primers were used to amplify the 8.15-kb and 8.30-kb dnd promoters. Fwd1: 5'-TGTCCAAACAGCTTGAAAAGTATCCATAGG-3' (for both promoter fragments); Rev1: 5'-CATCCATGTCTGGTACCGTCTGTGATGATG-3' (for 8.15-kb promoter); Rev2 5'-CTTTCAAGTGTCAAACCGACACACACAT-3' (for 8.30-kb promoter). A 0.98-kb 3' genomic fragment that contained the dnd translation stop codon and 3'UTR (nucleotides 172517 to 173493) was amplified by Fwd2 5'-TTTGCGGCCTTTCTAAGAATGTCAGAT-3' and Rev3: 5'-GCATTTTTATTGCCCATTTACCCTACCC-3'. Testicular genomic DNA was used for PCR amplification using Advantage ${ }^{\circledR}$ Genomic LA Polymerase Mix (Clontech). The genomic fragments were assembled to neo to generate

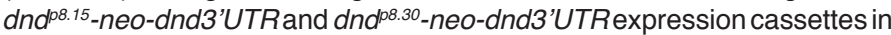
a modified Tol2pA vector (Villefranc et al., 2007) to enhance the insertion of the transgenic constructs (Kawakami, 2005). The resulting plasmids were designated pLIG330 for $d n d^{p 8.15}$-neo-dnd3'UTR and pLIG369 for $d n d^{p 8.30}$ neo-dnd3'UTR. To construct plasmid that expresses zebrafish Lif, the lif cDNA (Wong and Collodi, 2013) was sub-cloned into a plasmid in which the expression of zebrafish lif is driven by a CMV promoter (pLif, neo').

\section{Production of transgenic fish}

To produce transgenic fish, 1 to $2 \mathrm{nl}$ of a solution containing $7.5 \mathrm{ng} / \mu \mathrm{l}$ Tol 2 mRNA and $25 \mathrm{ng} / \mu \mathrm{l}$ of either pLIG330 or pLIG369 was injected into one to two-cell stage embryos. The embryos were raised to adults and sperm was collected from each male founder and analyzed by PCR for dnd-neo using dnd forward primer Fwd3: 5'-TTTTCCACCAATTTACAGGTGTGTCT-3' and neo reverse primer Rev4: 5'-ATACTTTCTCGGCAGGAGCA-3'. Three transgenic (dnd-neo positive) male founders from each line were chosen for further analysis and out-crossing to wild-type females to produce the stable transgenic lines.

\section{Production of feeder cells}

Astromal cell line, RTS34st, isolated from a rainbow trout spleen cell line RTS34 (Ganassin and Bols, 1999) was used as feeder cells in this study. RTS34st cells were cultured in Leibowitz's L-15 medium (Sigma-Aldrich) supplemented with $10 \%$ FBS (Harlan Lab) at $18^{\circ} \mathrm{C}$. To prepare RTS34stLif feeder cells, RTS34st cells were incubated and electroporated with linearized pLif plasmid using GenePulser Xcell electroporator (Bio-Rad). Cells were then selected with $500 \mu \mathrm{g} / \mathrm{ml}$ of antibiotic G418 to establish the RTS34st-Lif feeder cell line. The feeder lines were also transfected with plasmid that carries neo and/or pac expression cassette to generate 
antibiotic-resistant feeder lines. Conditioned medium was collected from confluent cultures every 3 days and stored at $-20^{\circ} \mathrm{C}$ before use.

\section{Immunocytochemistry}

Zebrafish gonads were fixed with $4 \%$ paraformaldehyde in phosphate buffered saline (PBS) for paraffin section. The serial sections $(5 \mu \mathrm{m})$ of gonads were used for immunocytochemical staining according to a published protocol (Wong and Zohar, 2004). Mouse monoclonal IgG against neomycin phosphotransferase II (Abcam) and rabbit antiserum against zebrafish Vasa (Knaut et al., 2000) were used together with Су3 AffiniPure goat anti-rabbit IgG and Alexa Fluor 488 AffiniPure goat anti-mouse IgG (Jackson ImmunoResearch Lab, Inc.) to visualize $\mathrm{Neo}$ and Vasa within the ovarian tissues of $T g(d n d$ :neo- $d n d)$ using a Nikon Eclipse TE200 fluorescence microscope (Nikon, Tokyo, Japan) equipped with a RT Slider digital camera (Spot Imaging Solution).

\section{Ovarian germ cell isolation and culture}

Ovaries combined from 8 to 10 female $T g$ (dnd:neo- $d n d$ ) zebrafish were minced and dissociated with $0.2 \%$ collagenase (Invitrogen). The resulting ovarian cell suspension was filtered through a $60-\mu \mathrm{m}$ mesh followed by a $37-\mu \mathrm{m}$ mesh and partially purified using a discontinuous Percoll (GE Healthcare) gradient (20\% and $40 \%$ ) according to a published protocol (Wong et al., 2011). The cell fraction was re-suspended in LDF medium (Collodi et al., 1992) and transferred into a plate that contained a confluent monolayer of growth-arrested feeder cells. The supplements were added at the final concentration of $5 \%$ FBS, $50 \mu \mathrm{g}$ protein $/ \mathrm{ml}$ zebrafish embryo extract, $1 \%$ heat treated trout serum (East Coast Biologics), $10 \mu \mathrm{g} / \mathrm{ml}$ of bovine insulin (Sigma-Aldrich), $20 \mathrm{ng} / \mathrm{ml}$ of human EGF (Invitrogen), 20 $\mathrm{ng} / \mathrm{ml}$ of FGF2 (Invitrogen) and $30 \%$ feeder cell conditioned medium. The cultures were maintained at $22{ }^{\circ} \mathrm{C}$ with medium changed every 3 or 4 days. After 2 to 4 months of drug selection, ovarian germ cells were further transfected with plasmid that encoded a Pac-DsRed fusion protein driven by a CMV promoter and selected with $2 \mu \mathrm{g} / \mathrm{ml}$ of antibiotic puromycin to establish a line of DsRed expressing ovarian germ cells.

\section{Growth assay}

$2 \times 10^{4}$ ovarian germ cells that also express DsRed were seeded into individual wells of a 6-well plate containing a confluent monolayer of growth-arrested RTS34st-Lif or RTS34st feeders. After 6 days of culture, ovarian germ cells in each individual well were collected by trypsinization, stained with trypan blue and counted using a hemacytometer and a fluorescence microscope.

\section{RNA extraction and RT-PCR analysis}

Total RNA was prepared from tissue or cultured ovarian germ cells using Trizol reagent (Invitrogen) followed by DNase treatment (Ambiom). The CDNA was synthesized using MMLV-RT (Promega) according to manufacturer's instructions. The sequences of the primers used for RT-PCR analysis were: vasa (Fwd4: 5'-TGTGGACGTGAGTGGCAGCAATC-3'/ Rev5: 5'-CTAGATAGCGCACTTTACTCAGG-3'), ßactin (Fwd5: 5'-AGACATCAGGGTGTCATGGTTGGT-3'/Rev6: 5'-TGGTCTCGTGGATACCGCAAGATT-3'); dnd (Fwd6: 5'-TCTGCAGGAATGGATGCAGAGGAA-3'/ Rev7: 5'-TCTGACGGTGATGGAAATGCCGTA-3') and nanos3 (Fwd7: 5'-CAGCATGGCTTTTTCTCTTCTC-3'/Rev8:5'-TCACCATGTTGATTTGGCGTA-3').

\section{Ovarian germ cell transplantation and analysis}

Cultured ovarian germ cells were trypsinized, washed and re-suspended in $50 \mu \mathrm{l}$ of L-15 medium, and immediately transplanted into two-week-old infertile zebrafish larvae treated with $d n d$ antisense morpholino (Slanchev et al., 2005) using a published method (Wong et al., 2011). Four weeks after transplantation, all the recipients were examined and 3DsRed-positive recipients were euthanized, and the status of the transplanted ovarian germ cells and the developmental stage of the gonads were evaluated. To determine if the transplanted cells were able to generate functional gametes the recipient fish were raised to sexual maturity and paired with wild-type zebrafish mates.

\section{Promoter and statistical analysis}

The promoter analysis of $d n d$ was done using SIGSCAN Version 4.05 suite of programs developed by Dr. Dan Prestridge (web version). Data obtained from growth assays were presented as the mean and standard deviation. For statistical analysis Student $t$ tests were applied. The significance was accepted at $p<0.05$.

\section{Acknowledgments}

We thank Dr. Holger Knaut for antiserum against zebrafish Vasa.

\section{References}

COLLODI, P., KAMEI, Y., SHARPS, A., WEBER, D. and BARNES, D. (1992). Fish embryo cell cultures for derivation of stem cells and transgenic chimeras. $\mathrm{Mol}$ Mar Biol Biotechnol 1: 257-265.

GANASSIN, R.C. and BOLS, N.C. (1999). A stromal cell line from rainbow trout spleen, RTS34ST, that supports the growth of rainbow trout macrophages and produces conditioned medium with mitogenic effects on leukocytes. In vitro Cell Dev Biol Anim 35: 80-86.

HANINGTON, P.C., PATTEN, S.A., REAUME, L.M., WASKIEWICZ, A.J., BELOSEVIC, M. and ALI, D.W. (2008). Analysis of leukemia inhibitory factor and leukemia inhibitory factor receptor in embryonic and adult zebrafish (Danio rerio). Dev Biol 314: 250-260.

HONG, Y., LIU, T., ZHAO, H., XU, H., WANG, W., LIU, R., CHEN, T., DENG, J. and GUI, J. (2004). Establishment of a normal medakafish spermatogonial cell line capable of sperm production in vitro. Proc Natl Acad Sci USA 101: 8011-8016.

HORVAY, K., CLAUSSEN, M., KATZER, M., LANDGREBE, J. and PIELER, T. (2006). Xenopus Dead end mRNA is a localized maternal determinant that serves a conserved function in germ cell development. Dev Biol 291: 1-11.

KANATSU-SHINOHARA, M., OGONUKI, N., INOUE, K., MIKI, H., OGURA, A., TOYOKUNI, S. and SHINOHARA, T. (2003). Long-term proliferation in culture and germline transmission of mouse male germline stem cells. Biol Reprod69:612-616.

KAWAKAMI, K. (2005). Transposon tools and methods in zebrafish. Dev Dyn 234 244-254.

KAWASAKI, T., SAITO, K., SAKAI, C., SHINYA, M. and SAKAI, N. (2012). Production of zebrafish offspring from cultured spermatogonial stem cells. Genes Cells 17: 316-325.

KNAUT, H., PELEGRI, F., BOHMANN, K., SCHWARZ, H. and NUSSLEIN-VOLHARD, C. (2000). Zebrafish vasa RNA but not its protein is a component of the germ plasm and segregates asymmetrically before germline specification. J Cell Biol 149: 875-888

SAKAI, N. (2002). Transmeiotic differentiation of zebrafish germ cells into functional sperm in culture. Development 129: 3359-3365.

SLANCHEV, K., STEBLER, J., DE LA CUEVA-MENDEZ, G. and RAZ, E. (2005) Development without germ cells: the role of the germ line in zebrafish sex differentiation. Proc Natl Acad Sci USA 102: 4074-4079.

VILLEFRANC, J.A., AMIGO, J. and LAWSON, N.D. (2007). Gateway compatible vectors for analysis of gene function in the zebrafish. Dev Dyn 236: 3077-3087.

WEIDINGER, G., STEBLER, J., SLANCHEV, K., DUMSTREI, K., WISE, C., LOVELLBADGE, R., THISSE, C., THISSE, B. and RAZ, E. (2003). dead end, a novel vertebrate germ plasm component, is required for zebrafish primordial germ cell migration and survival. Curr Biol 13: 1429-1434.

WONG, T.T. and COLLODI, P. (2013). Effects of specific and prolonged expression of zebrafish growth factors, Fgf2 and Lif in primordial germ cells in vivo. Biochem Biophys Res Commun 430: 347-351.

WONG, T.T., SAITO, T., CRODIAN, J. and COLLODI, P. (2011). Zebrafish germline chimeras produced by transplantation of ovarian germ cells into sterile host larvae. Biol Reprod 84: 1190-1197.

WONG, T.T. and ZOHAR, Y. (2004). Novel expression of gonadotropin subunit genes in oocytes of the gilthead seabream (Sparus aurata). Endocrinology 145:5210-5220.

YANG, Y., CONTAG, C.H., FELSHER, D., SHACHAF, C.M., CAO, Y., HERZENBERG L.A. and TUNG, J.W. (2004). The E47 transcription factor negatively regulates 
CD5 expression during thymocyte development. Proc Natl Acad Sci USA 101: 3898-3902.

YOUNGREN, K.K., COVENEY, D., PENG, X., BHATTACHARYA, C., SCHMIDT, L.S., NICKERSON, M.L., LAMB, B.T., DENG, J.M., BEHRINGER, R.R., CAPEL, B. et al., (2005). The Ter mutation in the dead end gene causes germ cell loss and testicular germ cell tumours. Nature 435: 360-364

ZOU, K., YUAN, Z., YANG, Z., LUO, H., SUN, K., ZHOU, L., XIANG, J., SHI, L., YU, Q., ZHANG, Y. et al., (2009). Production of offspring from a germline stem cell line derived from neonatal ovaries. Nat Cell Biol 11: 631-636.

\section{Further Related Reading, published previously in the Int. J. Dev. Biol.}

The fate of granulosa cells following premature oocyte loss and the development of ovarian cancers

Janet L. Pitman, Alan S. McNeilly, Judy R. McNeilly, Laura E. Hays, Grover C. Bagby Jr., Heywood R. Sawyer and Kenneth P. McNatty Int. J. Dev. Biol. (2012) 56: 949-958

Regulation of germ cell meiosis in the fetal ovary

Cassy M. Spiller, Josephine Bowles and Peter Koopman

Int. J. Dev. Biol. (2012) 56: 779-787

The Dr-nanos gene is essential for germ cell specification in the planarian Dugesia ryukyuensis Haruka Nakagawa, Hirotsugu Ishizu, Ayako Chinone,Kazuya Kobayashi and Midori Matsumoto Int. J. Dev. Biol. (2012) 56: 165-171

Differentiation of steroid-producing cells during ovarian differentiation in the protogynous Malabar grouper, Epinephelus malabaricus

Ryosuke Murata, Hirofumi Karimata, Yasuhisa Kobayashi, Ryo Horiguchi, Kazuo Kishimoto, Motofumi Kimura, Tohru Kobayashi, Kiyoshi Soyano and Masaru Nakamura

Int. J. Dev. Biol. (2011) 55: 619-625

Gonad-stimulating substance-like molecule from the radial nerve of the sea cucumber Hideki Katow, Tomoko Katow and Akihiko Moriyama Int. J. Dev. Biol. (2009) 53: 483-491

The allocation and differentiation of mouse primordial germ cells

T E Tsang, P L Khoo, R V Jamieson, S X Zhou, S L Ang, R Behringer and P P Tam Int. J. Dev. Biol. (2001) 45: 549-555

Female sterile mutations and egg chamber development in Drosophila Melanogaster S Gigliotti, D Rotoli, A Manzi, F Graziani and C Malva Int. J. Dev. Biol. (2000) 44: 581-589

Genomic sequence and embryonic expression of the zebrafish homeobox gene hox-3.4 $\mathrm{J} U$ Ericson, S Krauss and A Fjose Int. J. Dev. Biol. (1993) 37: 263-272
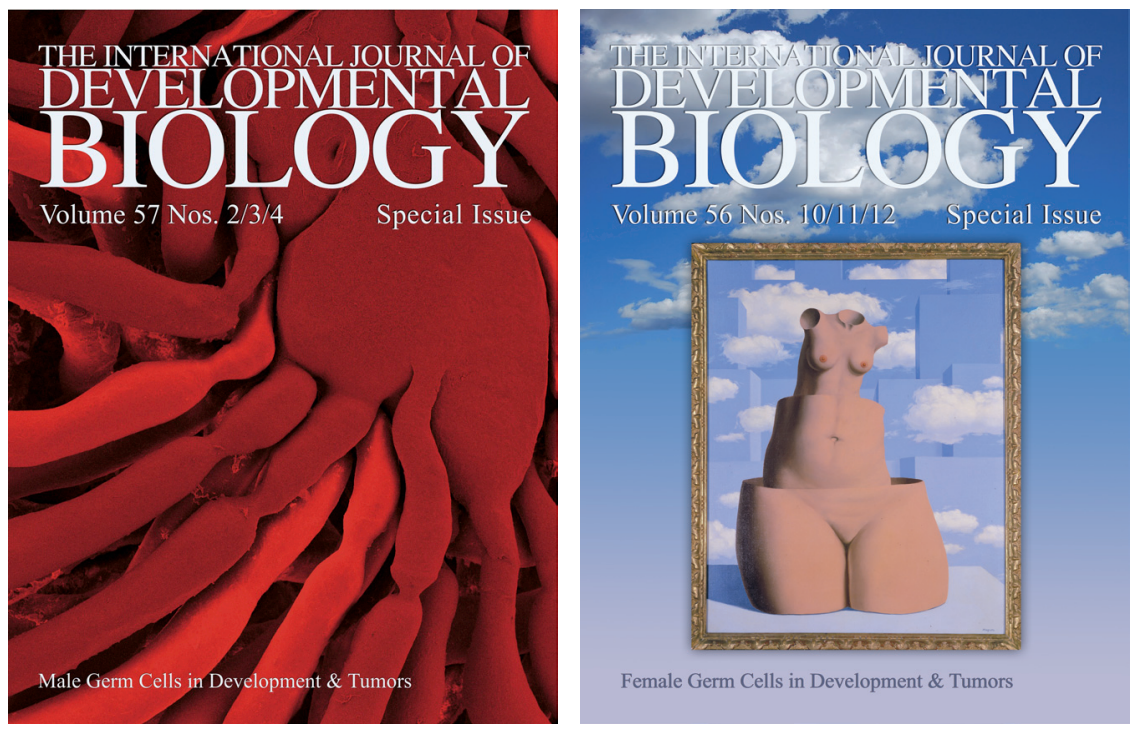
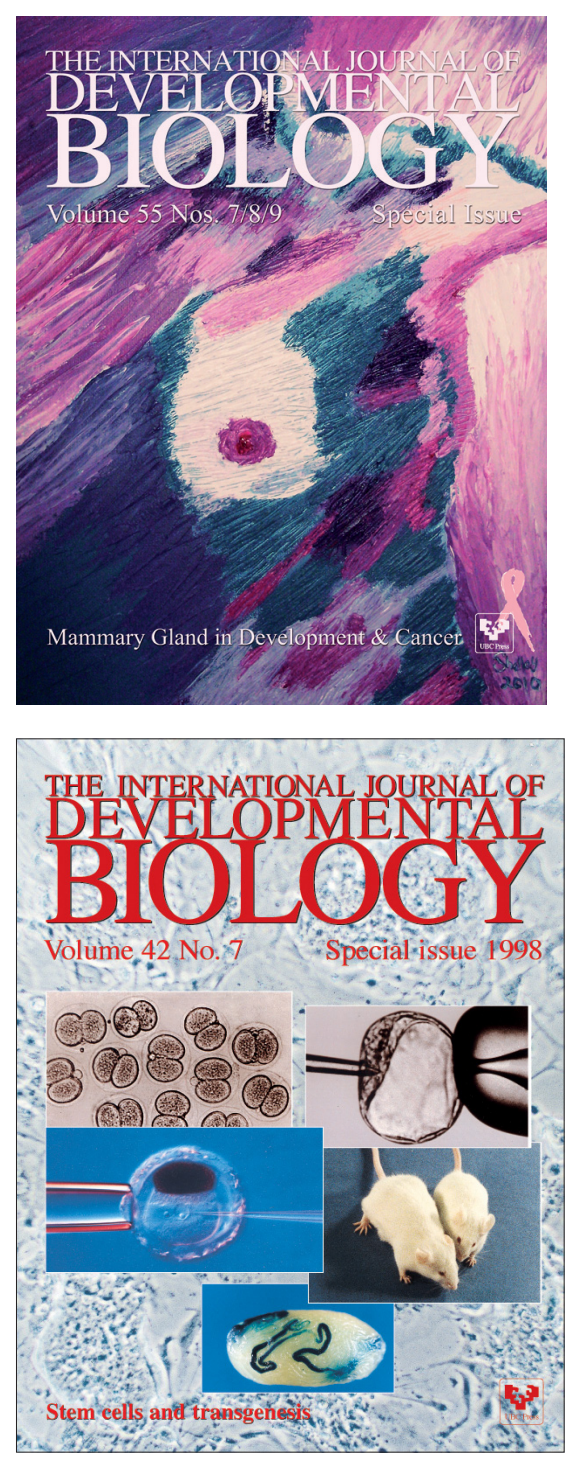